Sabine Skodda, MD

Wenke Grönheit, MD

Carsten Lukas, MD

Barbara Bellenberg, PhD

Sarah M. von Hein, MD

Rainer Hoffmann, MD

Carsten Saft, MD

Correspondence to

Dr. Saft:

carsten.saft@ruhr-uni-bochum.de

\title{
Two different phenomena in basic motor speech performance in premanifest Huntington disease
}

\section{ABSTRACT}

Objective: Dysarthria is a common feature in Huntington disease (HD). The aim of this crosssectional pilot study was the description and objective analysis of different speech parameters with special emphasis on the aspect of speech timing of connected speech and nonspeech verbal utterances in premanifest HD (preHD).

Methods: A total of 28 preHD mutation carriers and 28 age- and sex-matched healthy speakers had to perform a reading task and several syllable repetition tasks. Results of computerized acoustic analysis of different variables for the measurement of speech rate and regularity were correlated with clinical measures and MRI-based brain atrophy assessment by voxel-based morphometry.

Results: An impaired capacity to steadily repeat single syllables with higher variations in preHD compared to healthy controls was found (variance 1 : Cohen $d=1.46$ ). Notably, speech rate was increased compared to controls and showed correlations to the volume of certain brain areas known to be involved in the sensory-motor speech networks (net speech rate: Cohen $d=1.19$ ). Furthermore, speech rate showed correlations to disease burden score, probability of disease onset, the estimated years to onset, and clinical measures like the cognitive score.

Conclusions: Measurement of speech rate and regularity might be helpful additional tools for the monitoring of subclinical functional disability in preHD. As one of the possible causes for higher performance in preHD, we discuss huntingtin-dependent temporarily advantageous development processes of the brain. Neurology ${ }^{\circledR} 2016 ; 86: 1329-1335$

\section{GLOSSARY}

$\mathbf{5 y}$-prob $=5$-year probability of disease onset; $\mathbf{B A}=$ Brodmann area; COV1 = coefficient of variation for single syllable repetition; COV2 = coefficient of variation for repetition of pairs of syllables; DBS $=$ disease burden score; $\mathbf{G M}=$ gray matter; HD = Huntington disease; IntDur = interval duration; ISCED = International Standard Classification of Education score; maxSyIRep = maximum syllable repetition capacity; $\mathbf{N S R}=$ net speech rate; PD = Parkinson disease; $\mathbf{P R} \%=$ pause ratio; preHD = premanifest Huntington disease; syl = syllable; UHDRS-CS = Unified Huntington's Disease Rating Scale cognitive score; UHDRS-MS = Unified Huntington's Disease Rating Scale motor assessment; VBM = voxel-based morphometry; $\mathbf{V K T}=$ vowel keeping time; YOE = years of education; YTO = years to disease onset.

Dysarthria is a common symptom of Huntington disease (HD). ${ }^{1-4}$ These abnormalities have traditionally been attributed to involuntary sudden rapid and unpredictable movements of the speech muscles and disturbances in the accurate interaction of respiratory, laryngeal, velopharyngeal, and articulatory muscles. ${ }^{1}$ Moreover, since basal ganglia disorders in general have been found to affect the proper initiation, scaling, and maintenance of overlearned, automated basic motor programs essential for speech production, ${ }^{5,6}$ one would expect abnormalities of timing and temporal organization of speech in HD. ${ }^{7-10}$ Accordingly, in manifest HD there is a typical pattern of dysarthria with reduced articulation rates, bradykinetic articulatory movements with normal movement amplitudes, lower syllable reproduction rates with prolonged syllable durations, or an increased variability of utterance durations, based upon speech and nonspeech vocal tasks. ${ }^{11-16}$ Nothing, however, is known so far about the prevalence and pattern of these abnormal features in the premanifest stage of HD.

METHODS A total of 28 HD mutation carriers (premanifest HD [preHD]) with an average CAG repeat length of 42 (range 38-48, SD 2.2; 12 with paternal transmission, 16 with maternal transmission) as well as age- and sex-matched controls were recruited for this pilot study in our center (mean age 37.8 years, range 23-55 years; 14 male for the preHD group). There were no differences regarding

From the Department of Neurology, Knappschaftskrankenhaus (S.S., W.G.), and the Department of Radiology (C.L., B.B.) and the Department of Neurology, Huntington-Centre NRW (S.M.v.H., R.H., C.S.), St. Josef Hospital, Ruhr-University of Bochum, Germany.

Go to Neurology.org for full disclosures. Funding information and disclosures deemed relevant by the authors, if any, are provided at the end of the article.
Supplemental data at Neurology.org 
the years of education (YOE, $p=0.2)$ or the International Standard Classification of Education score (ISCED, $p=4.0$ ) between preHD and controls (mean YOE 15.4, SD 3.2 [range 10-23] vs 14.4, SD 2.3 [range 11-19] years; mean ISCED 3.4, SD 1.1 [range 2-5] vs 3.2 , SD 1 [range 2-6]). One participant from our control group had an education as a singer. Classification as preHD was based on expert raters' assessments of motor signs using the Unified Huntington's Disease Rating Scale motor assessment (UHDRSMS; diagnostic confidence level <4; mean UHDRS-MS score 2.18, SD 2.36, range 0-8). ${ }^{17-19}$ The dysarthria item of the UHDRS-MS was 0 in all participants. The neuropsychological tasks verbal fluency, symbol digit modalities, Stroop color naming, Stroop word reading, and Stroop interference performance were summarized as cognitive score (UHDRS-CS; mean 336.50, SD 43.85, range 242-411). ${ }^{19}$ In addition, simple (tapping) and complex (pegboard) fine motor skills were measured. ${ }^{10,20}$ The disease burden score (DBS) was calculated (mean 251.34, SD 81.84 , range $115.5-412.5),{ }^{21}$ as well as the estimated years to disease onset (YTO; mean 16.87, SD 8.3, range 5.28-36.41) and the 5 -year probability of disease onset (5y-prob; mean $16.4 \%$, SD 17.69 , range $0.7 \%-60.1 \%$ ). ${ }^{22}$ Three preHD participants had a history of depression, but were stable at the time of examination; none of the speakers was on psychotropic medication. None of the participants had manifest cognitive symptoms. As control group, we tested a group of 28 healthy persons matched by age and sex (mean age 40.82 years, range $20-56$ years; 14 male). All participants were native German speakers.

Speech samples were digitally recorded using commercial audio software (Steinberg WaveLab, Hamburg, Germany) and a headset microphone (Plantronics Audio 550 DSP, Santa Cruz, CA) with a sample rate of $44,100 \mathrm{~Hz}$. The speech task consisted of 4 subtests, which are described in detail in a previous publication of our group. ${ }^{16,23,24}$

Test 1: Reading task. Analysis of speech rate was performed by measuring the duration (in milliseconds) of each syllable (syl) and each pause, respectively, based on the oscillographic sound pressure signal (net speech rate [NSR], total speech time minus total pause time in syl/s) and pause ratio ( $\mathrm{PR} \%$, pause time percentage of total speech time). ${ }^{25}$

Test 2: Sustained phonation. Participants had to produce the vowel $/ \alpha /$ as long as possible with one single breath (vowel keeping time [VKT], in milliseconds).

Test 3: Maximum syllable repetition capacity. Participants had to reiterate the syllables /pa/, /ta/, /pa-ti/, and/pa-ko/ as fast as possible for at least 5 seconds (maximum syllable repetition capacity [maxSylRep], in syllables per second).

Test 4: Steadiness of syllable repetition. Participants had to repeat one single syllable (first trial: /pa/, second trial: /ta/) and a pair of syllables (first trial: /pa-ti/, second trial: /pa-ko/) in a comfortable self-chosen steady pace without acceleration or slowing articulatory speed. ${ }^{24}$ Stability of pace was defined as relative coefficient of variation for single syllable repetition (COV1) and repetition of pairs of syllables (COV2). Furthermore, the interval duration (IntDur, in milliseconds) described the duration from initiation of one utterance until the subsequent one.

Brain MRI data were acquired on a $1.5 \mathrm{~T}$ scanner for sagittal high-resolution T1-weighted 3D MRI data (magnetizationprepared rapid gradient echo) in 24 preHD participants for voxel-based morphometry (VBM) as described earlier. ${ }^{26}$

Voxelwise statistics used multiple regression analyses of gray matter (GM) including the parameters that were found to be different from controls (NSR, maxSylRep, COV1, and COV2) as covariates and age as confounder. Cluster locations in which GM was associated with the covariates $(p<0.001)$ were identified using common anatomical atlases. ${ }^{26}$

Unpaired $t$ test for independent samples, Pearson correlation, and Spearman rank test were used for statistical analysis of normal and not normal distributed data. According to the exploratory approach of the study, data were not adjusted for multiple testing. In an additional exploratory analysis, preHD subgroups (defined by the median of YTO) for participants far from ( $>16.7$ years) and close to $(<16.7$ years $)$ disease onset were compared. Effect sizes were measured with Cohen $d$.

Standard protocol approvals, registrations, and patient consents. Our study was in compliance with the Helsinki Declaration and had been approved by the ethics committee of the Ruhr University Bochum. Written informed consent was obtained from each participant.

\section{RESULTS Comparison between the preHD group and} controls. PreHD speakers showed a specific pattern of speech alterations in the reading task, characterized by an increase of speech rate/NSR (6.05 \pm 0.62 vs $5.33 \pm 0.61 ; p<0.0001)$ without an altered proportion of speech pauses/PR\%.

Concerning nonspeech vocal performance (tests 3 and 4), preHD participants showed a clear increase of maxSylRep $(5.43 \pm 1.21$ vs $4.62 \pm 1.61, p=0.03)$, and a marked irregularity in the syllable repetition tasks, mirrored by an increased COV1 (1.61 \pm 0.54 vs $0.98 \pm 0.31, p<0.0001)$ and COV2 $(2.24 \pm 0.89$ vs $1.31 \pm 1.21, p=0.002)$. The self-chosen speed of repetition (IntDur) showed a tendency to higher pace, whereas VKT showed no differences between the preHD and the control group. Numerical data and statistical comparisons are summarized in table 1.

Correlations within the preHD group. Concerning the parameters of connected speech, NSR showed correlations with UHDRS-MS $(r=-0.476, p=0.005)$, the DBS $(r=-0.636, p=0.0001)$, the cognitive sum score $(r=0.554, p=0.001)$, with highest correlations with the color naming subtest $(r=0.610, p<0.001)$, the estimated YTO $(r=0.614, p=0.0003)$, and the $5 y$-prob $(r=-0.641, p=0.0001)$.

Of the measures of nonspeech vocal performance, only maxSylRep was correlated with UHDRS-MS $(r=-0.428, p=0.011)$, the DBS $(r=-0.425$, $p=0.012)$, the cognitive sum score $(r=0.484, p=$ $0.005)$, with highest correlations with the symbol digit modalities $(r=0.550, p=0.001)$ and color naming subtests $(r=0.455, p=0.007)$, YTO $(r=0.438, p=$ $0.011)$, 5y-prob $(r=-0.397, p=0.008)$, and the measures of motor performance (tapping: $r=0.528$, $p=0.002 /$ pegboard: $r=-0.415, p=0.014)$. A weak correlation was found between the number of CAG repeats and maxSylRep $(r=-0.357, p=0.03)$. The other syllable repetition parameters COV and IntDur showed no such correlations. 


\begin{tabular}{|c|c|c|c|c|}
\hline \multirow[t]{2}{*}{$\begin{array}{l}\text { Compe } \\
\text { group }\end{array}$} & \multicolumn{4}{|c|}{$\begin{array}{l}\text { omparisons of speech measures between the premanifest Huntington disease (preHD) and contro } \\
\text { roup }\end{array}$} \\
\hline & $\begin{array}{l}\text { preHD }(n=28) \\
14 \text { men, mean (SD) }\end{array}$ & $\begin{array}{l}\text { Controls }(n=28), \\
14 \text { men, mean (SD) }\end{array}$ & $p$ Value, $t$ test & $\begin{array}{l}\text { Effect size, } \\
\text { Cohen } d\end{array}$ \\
\hline Age & $37.79 / 8.55$ & $40.82 / 8.86$ & 0.2 & \\
\hline NSR, syl/s & $6.05 / 0.62$ & $5.33 / 0.61$ & $<0.0001$ & 1.19 \\
\hline PR\% & $14.18 / 3.43$ & $14.20 / 6.09$ & 0.9 & \\
\hline VKT, ms & $16,957 / 7,243$ & $16,524 / 8,320$ & 0.8 & \\
\hline maxSylRep, syl/s, Hz & $5.43 / 1.21$ & $4.62 / 1.61$ & 0.03 & 0.58 \\
\hline Cov1 & $1.61 / 0.54$ & 0.98/0.31 & $<0.0001$ & 1.46 \\
\hline cov2 & $2.24 / 0.89$ & $1.31 / 1.21$ & 0.002 & 0.89 \\
\hline IntDur, ms & $443 / 140$ & $530 / 222$ & 0.09 & 0.48 \\
\hline
\end{tabular}

Abbreviations: COV1 = relative coefficient of variation for single syllable repetition; COV2 = relative coefficient of variation for repetition of pairs of syllables; IntDur = interval duration; maxSylRep = maximum syllable repetition capacity; $\mathrm{NSR}=$ net speech rate (total speech time minus total pause time in syllables per second); PR\% = pause ratio (pause time percentage of total speech time); syl = syllable; VKT = vowel keeping time.

Comparisons within the preHD group for participants far from and close to disease onset. The additional exploratory comparison between 14 preHD participants far from onset of the disease ( $>16.7$ years; 5 male) and participants close to onset with an estimated onset of disease $<16.7$ years ( 9 male) revealed relevant differences concerning UHDRS-CS (far: 358 [SD 36] vs close: 315 [SD 41]; $p=0.008$; Cohen $d$ : 1.16 ) and pegboard (far: 41.8 [SD 3.51] vs close: 48.0 seconds [SD 10.1]; $p=0.045$; Cohen $d$ : 0.85 ), but also for NSR (far: 6.40 [SD 0.43] vs close: 5.70 [SD 0.59]; $p=0.002$; Cohen $d: 1.41$ ) and maxSylRep (far: 5.87 [SD 1.23] vs close: 4.98 [SD 1.05]; $p=0.05$; Cohen $d: 0.81$ ) and a trend to higher COV values in the close group (COV1 far: 1.58 [SD 0.50] vs close: 1.64 [SD 0.60]; $p=0.7$; Cohen $d: 0.11$; and COV2 far: 2.19 [SD 0.66] vs close: 2.29 [SD 1.08]; $p=0.8$; Cohen $d: 0.14)$. No differences were found for age, UHDRS-MS, tapping, VKT, or PR\% (results not shown). In NSR and COV1, there was a difference also in comparison with the accordant control subgroup $(p<0.01)$ for both subgroups; in COV2, only for the close subgroup. In maxSylRep, the difference in comparison with the accordant far control subgroup was $p<0.05$.

VBM: Correlations between speech and nonspeech vocal performance and brain GM. We found correlations with increased brain GM in an area in the cerebellar vermis (declive/vermal lobule VI) and NSR (figure 1). Correlations between maxSylRep and increased GM were detected in the left inferior frontal gyrus in an area assigned to Brodmann area (BA) 47 (figure 2). COV2 was correlated with GM atrophy in an area in the right primary somatosensory cortex (BA 3, BA 2), in another area in the left supramarginal gyrus

Figure 1 Correlations between higher net speech rate and increased cerebellar gray matter (GM) volume in the vermis

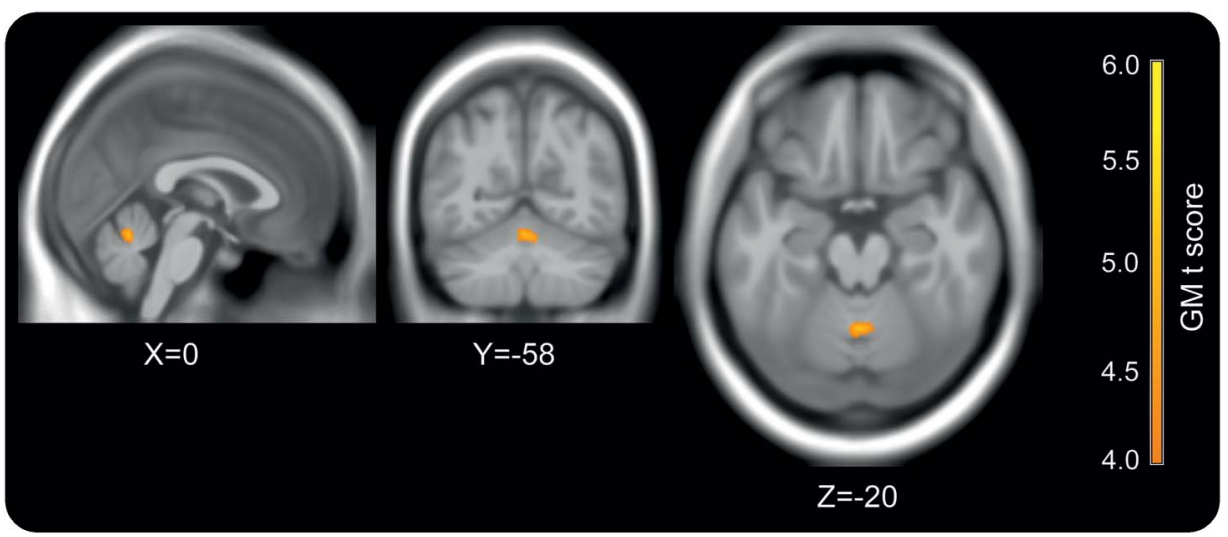

Age was used as a covariate of no interest. Uncorrected clusters (yellow) were collected at $p<0.001$ and depicted if cluster size was higher than 60 voxels. 


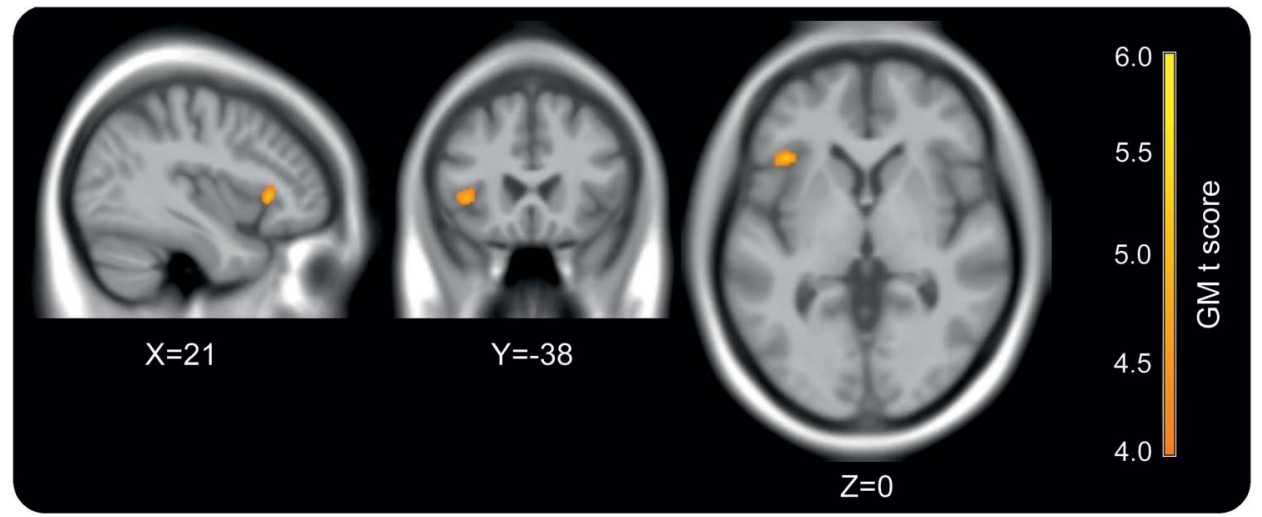

Age was used as a covariate of no interest. Uncorrected clusters (yellow) were collected at $p<0.001$ and depicted if cluster size was higher than 60 voxels.

(BA 40), and in the left precuneus (BA 7), a part of the secondary somatosensory cortex (figure 3). A volume decrease in these cortical areas, which all had somatosensory or sensory-motor functions, was associated with an increase in COV2 (Montreal Neurological Institute coordinates and voxel levels; see table e-1 on the Neurology ${ }^{\circledR}$ Web site at Neurology.org). We did not observe any correlations for COV1. According to the explorative character of the study, in a second approach we relaxed the significance threshold from $p<0.001$ to $p<0.005$ to assess the tendencies for additional significances between brain GM and the speech tests. Using this approach, we observed additional correlations between GM and maxSylRep bilaterally in the caudate nuclei (data not shown), which might become more evident in larger studies on preHD participants.

DISCUSSION According to our data, carriers of the HD mutation feature abnormalities of rate and regularity of connected speech and nonspeech vocal performance even in the early premanifest stage of the disease. Compared to healthy control speakers, they show an increased articulation rate (increased maximum syllable repetition capacity and net speech rate); however, the higher speaking rates are accompanied by imprecise pace performance, with higher variability in syllable repetition mirrored by increased COV. Thus, there are 2 different phenomena to observe in this study: (1) compared to controls, preHD showed a higher variability in speech and timing, with worsening in participants closer to onset of the disease; and (2) at the same time, they speak faster compared to controls, but with an approximation to the speech rate of controls in participants closer to onset of the disease.
Since speakers with manifest HD have been found to speak more slowly than healthy controls, the decline of articulatory speed seems to mirror the progression of the underlying pathophysiology. ${ }^{16}$

Steadiness and regularity of syllable repetition seem to be compromised already in the premanifest stage far from disease onset and also tend to deteriorate over time. In healthy speakers, the timely and rhythmic production of single syllables seems to depend on the integrity of subcortical networks including putamen, pallidum, and thalamus, parts of the cerebellum, and the left caudate nucleus. ${ }^{27-29}$ Consequently, abnormalities of steady syllable repetition have been found in different kinds of movement disorders, such as Parkinson disease, spinocerebellar ataxia, and recently also in manifest HD. ${ }^{16,23,30}$ Abnormalities of timing and temporal organization in manifest and premanifest HD were also described for other sensitive motor measures, like tapping. ${ }^{7-9}$ Interval timing and rhythmicity was described to be dependent on basal ganglia integrity. ${ }^{31,32}$ In accordance, a deterioration of timeestimation processes in manifest and even premanifest HD was described. ${ }^{33}$ Accordingly, instability of syllable repetition, in the current study, as indicated by elevated COV1 and COV2, with even more pronounced findings in our close-to-onset-subgroup, is at least partly a manifestation of basal ganglia dysfunction at this stage. However, the absence of correlations with clinical data and correlation with more somatosensory cortex areas in the voxel morphology analysis reveals that these processes are more complex and not only basal ganglia-dependent. It has been suggested that compensatory mechanisms help to maintain a constant speech rate by detecting errors between the internally predicted and the actually provided auditory information by timing- and 
Figure 3 Correlations between repetition of pairs of syllables (COV2) and somatosensory cortical areas

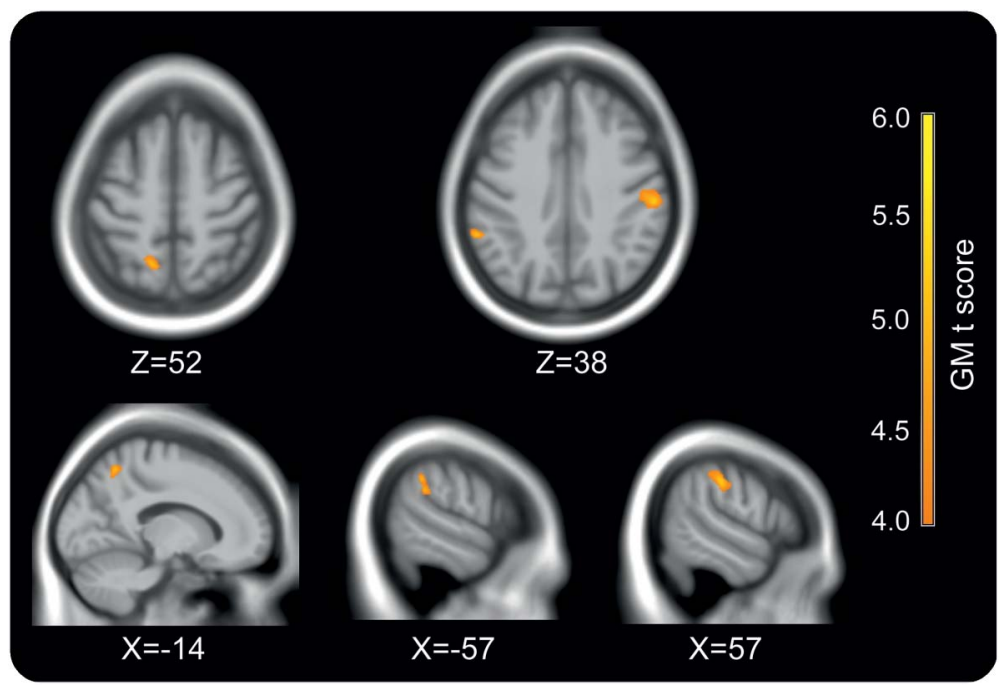

Correlations between irregularity of repetition of pairs of syllables (COV2) and atrophy of an area in the right primary somatosensory cortex (Brodmann area [BA] 3, BA 2), in another area in the left supramarginal gyrus (BA 40), and in the left precuneus (BA 7), a part of the secondary somatosensory cortex. Age was used as a covariate of no interest. Uncorrected clusters (yellow) were collected at $p<0.001$ and depicted if cluster size was higher than 60 voxels. $\mathrm{GM}=$ gray matter .

context-dependent effects of feedback alterations. ${ }^{29,34}$ Disturbances of such feedback mechanisms might explain the correlations between increased COV2 and atrophy in somatosensory areas found in our study.

The finding of faster vocal performance in the premanifest HD stage is surprising and difficult to interpret. At first sight, one might argue that participants at risk for $\mathrm{HD}$ could make more efforts to prove that their speech motor skills are unaffected. Subtle cognitive changes such as frontal lobe dysfunction with disinhibition phenomena could at least contribute to higher speech velocities, which is corroborated by the correlations between speech rate and the cognitive score, or accelerated speech rate might reflect a more hypokinetic dysarthria as described for Parkinson disease (PD). Unlike in PD, however, we observed no changes in volume or accelerated speech rate in the course of speaking, but a general increase in overall speaking rate as the first and isolated abnormality of speech.

However, those speech measures showed a clear correlation to parameters reflecting the progressive pathophysiology of HD, such as the disease burden score, probability, and years to onset, and were correlated with the volume of some distinctive cortical brain areas reported to be integral parts of the widespread speech-language brain network. ${ }^{35} \mathrm{Net}$ speech rate was correlated with the volume of a distinctive part of the cerebellar vermis (declive) and although the explicit function of the cerebellar declive in vocal performance is unknown, the cerebellum in general has been recognized as one part of the network involved in generation of appropriate motor commands on the syllable level..$^{27,29}$ The maximum syllable repetition showed a correlation to the volume of the left inferior frontal gyrus (BA 47 and 48, which are involved in language processing ${ }^{36}$ ) and-less clearly with $p<0.005$ - to the basal ganglia volume. These correlations favor the interpretation that faster syllable and higher speech rate are not caused by better motivation, but instead are the consequence of faster speech motor performance accompanied by higher volume of the accordant brain structures.

By contrast, most previous studies demonstrate a progressive brain atrophy in preHD, beginning approximately 15 years prior to disease onset. ${ }^{9,17}$ There are, however, also some rare reports on increased brain structure in preHD participants. Paulsen et al. ${ }^{37}$ refer to an increase of overall cerebral cortical volume in a group of 24 preHD participants with estimated years to onset of 16.92 and therefore similar to our group; however, no differentiation was made between different cortical areas. Since in our study only the preHD participants underwent MRI scanning, we cannot decide whether the correlation of increased speech rate compared to controls and correlation to certain brain areas are caused by an increase of volume or rather by a less pronounced local atrophy of these regions.

Therefore, one might only hypothesize that our preHD speakers were able to maintain and even exceed the demands of articulatory speed by an overactivation or dysfunctional hypercompensation, while the maintenance of a stable pace (which reflects more automated basic motor speech performance) is nonetheless impaired since it rather relies on the integrity of basal ganglia. The possibility of a temporarily enhanced activity and function in the very early premanifest stage of the disease, however, had also been described in a transgenic rat model of HD and in a recent published PET study investigating cerebral adenosine A1 receptors in HD. ${ }^{38,39}$ For the transgenic rat model, the authors reported biphasic motor behavior with faster and better motor performance in very young premanifest animals followed by a slow decline and resulting in an impaired motor function over time..$^{38}$ The possibility of an at least temporarily enhanced function in the very early premanifest stage, and thus a new to define phase in the course of the disease, was already discussed earlier and might reflect a part of the disease cascade, or could also be caused by developmental processes, or even evolutionary advantages. ${ }^{18,40}$ In this context, Lo Sardo et al. ${ }^{40}$ found that huntingtin is essential for homotypic interactions between neuroepithelial cells in deuterostomes. They discussed that the progressive increase in the length of 
its CAG repeat sequence in deuterostomes may be implicated in the evolutionary path that has led to the acquisition of morphologic complex nervous systems. Our findings would extend this new approach for HD in a time-dependent, initially advantageous, most likely developmental effect with increased performance that is detectable only very early in life, before the known adverse effects of this mutation occur.

Taking into account our earlier study on speech in manifest $\mathrm{HD}$, one could suggest a specific pattern and time course of speech abnormalities with elevated articulatory speed in the period far from disease onset that declines over time and becomes slower and more dysrhythmic than in healthy controls in the manifest stages of HD. ${ }^{16}$

Difference of articulation rate showed a higher effect size in our study than motor and cognitive tasks, and thus they might be even more sensitive than monitoring of disease progression tasks described so far. ${ }^{9,17}$ Especially the tasks of nonspeech vocal performance applied in our study are expected to be language-independent; furthermore, the speech investigations are noninvasive, inexpensive, and easy to apply. Therefore, it might be worthwhile to survey their usefulness in international multicenter studies.

\section{AUTHOR CONTRIBUTIONS}

Study concept and design: Dr. Skodda, Dr. Grönheit, Dr. Lukas, Dr. Bellenberg, Dr. Saft. Acquisition of data: Dr. Saft, S.M. von Hein, Dr. Hoffmann, Dr. Lukas. Analysis and interpretation of data: Dr. Skodda, Dr. Bellenberg, Dr. Lukas, Dr. Saft. Statistical analysis: Dr. Skodda, Dr. Bellenberg, Dr. Saft. Drafting of manuscript: Dr. Skodda, Dr. Saft. Critical revision of the manuscript for important intellectual content: Dr. Grönheit, Dr. Lukas, Dr. Bellenberg, S.M. von Hein, Dr. Hoffmann. Administrative, technical, and material support: Dr. Skodda, Dr. Bellenberg.

\section{ACKNOWLEDGMENT}

The authors thank the participants and their families for their time and interest in this study.

\section{STUDY FUNDING}

No targeted funding reported.

\section{DISCLOSURE}

S. Skodda has received honoraria and travel or accommodation payments from Teva, Medtronic, St. Jude Medical, Abbvie, Desitin, UCB, and Orion. W. Grönheit has received travel or accommodation payment or participation fee from UCB, Eisai, Desitin, Abbot, and Boehringer Ingelheim. C. Lukas has received consulting and speaker's honoraria from BiogenIdec, Bayer Schering, Novartis, Sanofi, Genzyme, and Teva, and has received research scientific grant support from Bayer Schering, Teva, and MerckSerono. B. Bellenberg has received speaker's honoraria and research grant support from Bayer Healthcare. S. von Hein and R. Hoffmann report no disclosures relevant to the manuscript. C. Saft reports grants from Teva Endowed Professorship, grants from Cure Huntington's Disease Initiative (CHDI), grants from Biogen, and personal fees from Temmler Pharma GmbH \& Co. KG and Desitin Arzneimittel GmbH, outside the submitted work; and he received institutional compensation and/or travel or accommodation payments in the context of the Registry Study (Euro HD Network), the ENROLL study (CHDI), the MitoNet study, the ACR16 Study (Neurosearch), the AFQ
Study (Novartis), the Selisistat Studies (Siena Biotech), the PRIDE HD and LEGATO HD Study (TEVA), ISIS Pharmaceuticals, and the Amaryllis Study (Pfizer). Go to Neurology.org for full disclosures.

Received October 9, 2015. Accepted in final form January 4, 2016

\section{REFERENCES}

1. Roos RA. Huntington's disease: a clinical review. Orphanet J Rare Dis 2010;5:40.

2. Rusz J, Klempir J, Baborova E, et al. Objective acoustic quantification of phonatory dysfunction in Huntington's disease. PloS One 2013;8:e65881.

3. Velasco Garcia MJ, Cobeta I, Martin G, AlonsoNavarro H, Jimenez-Jimenez FJ. Acoustic analysis of voice in Huntington's disease patients. J Voice 2011;25: 208-217.

4. Darley FL, Aronson AE, Brown JR. Differential diagnostic patterns of dysarthria. J Speech Hear Res 1969;12: 246-269.

5. Abbs JH, Gracco VL, Cole KJ. Control of multimovement coordination: sensorimotor mechanisms in speech motor programming. J Mot Behav 1984;16:195-231.

6. Ramig LA. Acoustic analyses of phonation in patients with Huntington's disease: preliminary report. Ann Otol Rhinol Laryngol 1986;95:288-293.

7. Bechtel N, Scahill RI, Rosas HD, et al. Tapping linked to function and structure in premanifest and symptomatic Huntington disease. Neurology 2010;75:2150-2160.

8. Rowe KC, Paulsen JS, Langbehn DR, et al. Self-paced timing detects and tracks change in prodromal Huntington disease. Neuropsychology 2010;24:435-442.

9. Tabrizi SJ, Langbehn DR, Leavitt BR, et al. Biological and clinical manifestations of Huntington's disease in the longitudinal TRACK-HD study: cross-sectional analysis of baseline data. Lancet Neurol 2009;8:791-801.

10. Saft C, Andrich J, Meisel NM, Przuntek H, Muller T. Assessment of simple movements reflects impairment in Huntington's disease. Mov Disord 2006;21:1208-1212.

11. Ackermann H, Hertrich I, Daum I, Scharf G, Spieker S. Kinematic analysis of articulatory movements in central motor disorders. Mov Disord 1997;12:1019-1027.

12. Ackermann H, Hertrich I, Hehr T. Oral diadochokinesis in neurological dysarthrias. Folia Phoniatr Logop 1995;47: 15-23.

13. Hertrich I, Ackermann H. Acoustic analysis of speech timing in Huntington's disease. Brain Lang 1994;47: 182-196.

14. Ludlow CL, Connor NP, Bassich CJ. Speech timing in Parkinson's and Huntington's disease. Brain Lang 1987; 32:195-214.

15. Volkmann J, Hefter H, Lange HW, Freund HJ. Impairment of temporal organization of speech in basal ganglia diseases. Brain Lang 1992;43:386-399.

16. Skodda S, Schlegel U, Hoffmann R, Saft C. Impaired motor speech performance in Huntington's disease. J Neural Transm 2014;121:399-407.

17. Paulsen JS, Long JD, Ross CA, et al. Prediction of manifest Huntington's disease with clinical and imaging measures: a prospective observational study. Lancet Neurol 2014;13:1193-1201.

18. Saft C. What is the course of Huntington's disease? Lancet Neurol 2014;13:1165-1166.

19. Huntington Study Group. Unified Huntington's Disease Rating Scale: reliability and consistency. Mov Disord 1996;11:136-142. 
20. Saft C, Andrich J, Meisel NM, Przuntek H, Muller T. Assessment of complex movements reflects dysfunction in Huntington's disease. J Neurol 2003;250:1469-1474.

21. Penney JB Jr, Vonsattel JP, MacDonald ME, Gusella JF, Myers RH. CAG repeat number governs the development rate of pathology in Huntington's disease. Ann Neurol 1997;41:689-692.

22. Langbehn DR, Brinkman RR, Falush D, Paulsen JS, Hayden MR. A new model for prediction of the age of onset and penetrance for Huntington's disease based on CAG length. Clin Genet 2004;65:267-277.

23. Skodda S, Schlegel U. Speech rate and rhythm in Parkinson's disease. Mov Disord 2008;23:985-992.

24. Skodda S, Flasskamp A, Schlegel U. Instability of syllable repetition as a marker of disease progression in Parkinson's disease: a longitudinal study. Mov Disord 2011;26:59-64.

25. Boersma P, Weenik D. PRAAT: a system for doing phonetics by computer. Report of the Institute of Phonetic Sciences of the University of Amsterdam [online]. Available at: www.fon.hum.uva.nl/praat/. Accessed in 2013.

26. Bellenberg B, Schneider R, Weiler F, et al. Cervical cord area is associated with infratentorial grey and white matter volume predominantly in relapsing-remitting multiple sclerosis: a study using semi-automated cord volumetry and voxel-based morphometry. Mult Scler Relat Disord 2015;4:264-272.

27. Wildgruber D, Ackermann H, Grodd W. Differential contributions of motor cortex, basal ganglia, and cerebellum to speech motor control: effects of syllable repetition rate evaluated by fMRI. NeuroImage 2001;13:101-109.

28. Bohland JW, Guenther FH. An fMRI investigation of syllable sequence production. NeuroImage 2006;32:821-841.

29. Penhune VB, Zattore RJ, Evans AC. Cerebellar contributions to motor timing: a PET study of auditory and visual rhythm reproduction. J Cogn Neurosci 1998;10:752-765.

30. Schmitz-Hubsch T, Eckert O, Schlegel U, Klockgether T, Skodda S. Instability of syllable repetition in patients with spinocerebellar ataxia and Parkinson's disease. Mov Disord 2012;27:316-319.

31. Bartolo R, Merchant H. Beta oscillations are linked to the initiation of sensory-cued movement sequences and the internal guidance of regular tapping in the monkey. J Neurosci 2015;35:4635-4640.

32. Dhamala M, Pagnoni G, Wiesenfeld K, Zink CF, Martin M, Berns GS. Neural correlates of the complexity of rhythmic finger tapping. Neuroimage 2003;20:918-926.

33. Beste C, Saft C, Andrich J, Muller T, Gold R, Falkenstein M. Time processing in Huntington's disease: a group-control study. PLoS One 2007;2:e1263.

34. Mochida T, Gomi H, Kashino M. Rapid change in articulatory lip movement induced by preceding auditory feedback during production of bilabial plosives. PLoS One 2010;5:e13866.

35. Fuertinger S, Horwitz B, Simonyan K. The functional connectome of speech control. PLoS Biol 2015;13: e1002209.

36. Haller S, Klarhoefer M, Schwarzbach J, Radue EW, Indefrey P. Spatial and temporal analysis of fMRI data on word and sentence reading. Eur J Neurosci 2007;26: 2074-2084.

37. Paulsen JS, Magnotta VA, Mikos AE, et al. Brain structure in preclinical Huntington's disease. Biol Psychiatry 2006; 59:57-63.

38. Nguyen HP, Kobbe P, Rahne H, et al. Behavioral abnormalities precede neuropathological markers in rats transgenic for Huntington's disease. Hum Mol Genet 2006;15:3177-3194.

39. Matusch A, Saft C, Elmenhorst D, et al. Cross sectional PET study of cerebral adenosine A(1) receptors in premanifest and manifest Huntington's disease. Eur J Nucl Med Mol Imag 2014;41:1210-1220.

40. Lo Sardo V, Zuccato C, Gaudenzi G, et al. An evolutionary recent neuroepithelial cell adhesion function of huntingtin implicates ADAM10-Ncadherin. Nat Neurosci 2012;15:713-721.

\section{BrainPAC}

BrainPAC is the American Academy of Neurology's (AAN) federal political action committee.

- Since its inception, more than 3,000 AAN members have contributed $\$ 1,800,000$ to BrainPAC.

- BrainPAC contributed $\$ 620,000$ to candidates running for Congress in 2014.

- During the 2014 congressional campaign, 89 percent of candidates supported by BrainPAC won their elections.

BrainPAC supports both Democrats and Republicans who support issues important to the practice of neurology and the care of patients with neurologic conditions. US AAN members are invited to learn more at BrainPAC.org. 